\title{
Computing the distance to instability for delay systems with uncertainties in the system matrices and in the delay terms
}

\author{
Francesco Borgioli ${ }^{1}$ and Wim Michiels ${ }^{1}$
}

\begin{abstract}
In this paper we propose an algorithm to compute the distance to instability of a linear system of delay differential equations (DDEs) containing uncertainties in the delay terms as well as in matrices coefficients. For what regards the system matrices, any structure on the perturbation can be considered in order to allow only specific parameters to change; moreover, real-valued matrix perturbations are taken into account. The algorithm relies on the computation of the pseudospectral abscissa of the system and performs a bisection-Newton's method to find the minimum size of the perturbation that generates instability. A few illustrative examples, including a model for a rotating cutting machine, finally show the correctness and the efficiency of the method.
\end{abstract}

\section{INTRODUCTION}

In the investigation of a dynamical system's asymptotical behaviour, the computation of the distance to instability (or stability radius) plays a fundamental role in the assessment of its stability robustness. Many existing algorithms allow to compute the stability radius of dynamical systems described by first or higher order ODEs as, among others, the ones presented in [1], [2] and [3]. However, when we come to delay differential equations (DDEs), the analysis of stability and its robustness becomes a much more complex problem. In this paper we deal with systems of DDEs as follows

$$
\dot{x}(t)=\sum_{i=1}^{m} A_{i} x\left(t-\tau_{i}\right),
$$

where $x(t) \in \mathbb{R}^{n}$ is the state variable, $A_{1}, \ldots, A_{m} \in$ $\mathbb{R}^{n \times n}, n \geq 2$ and $0 \leq \tau_{1}<\cdots<\tau_{m}$ are the timedelays. The solution of this system is asymptotically stable if and only if all the roots of the characteristic equation of the associated delay eigenvalue problem (DEP) lie in the open left-half of the complex plane, namely they all have a negative real part. In literature, many attempts to characterize the asymptotical behaviour of a dynamical system in the delayparameter space have been made: without being exhaustive, several characterizations of delay-dependent stability (see [4] and [5]) and delay-independent stability (see [6] and [7]) have been proposed. We refer the reader interested in a complete overview of the methods to the monograph [8]. Less results are available when we consider the robustness of the stability w.r.t. potential uncertainties in our mathematical model: in [9], the robustness of the delay-independent stability is assessed w.r.t. uncertainties in system matrices; in [10] a lower-bound on the stability radius of a time-delay system simultaneously subject to real-valued perturbations

\footnotetext{
${ }^{1}$ Department of Computer Science, KU Leuven, Celestijnenlaan 200A, 3001 Heverlee, Belgium, name. surname@cs.kuleuven. be
}

on the delay terms and to complex-valued perturbations on the system matrices is presented.

In this paper, we consider and fully exploit structured real-valued perturbations of the system matrices and realvalued perturbations on the delay terms: this is the most realistic framework to investigate the robustness of stability. Here we propose a method to exactly compute the distance to instability of a time-delay system as in (1) w.r.t. to the aforementioned uncertainties on the system. The distance to instability is defined as the size of the smallest perturbation that pushes one (or more) eigenvalues of a stable system on the imaginary axis, thus generating instability; therefore, by definition, the distance to instability is strictly connected with the pseudospectral abscissa, that is the real part of the rightmost eigenvalue of the systems arisen from all the possible $\varepsilon$-bounded perturbations on the original system. As we shall see in Section II, the distance to instability as the unique root of equation can also be defined as the minimum perturbation for which the system is not robustly stable.

In literature, many existing algorithms are available to compute the pseudospectral abscissa, both for linear and nonlinear eigenvalue problems (e.g. polynomial or delay eigenvalue problem); among the others, the method developed in [11] accounts for real-valued perturbations in the standard eigenvalue problem, whereas in [12] complexvalued uncertainties on the matrices coefficients of nonlinear eigenvalue problems are considered. The real-valued nature of uncertainties and the nonlinearity of the eigenvalue problem are simultaneously included in the method developed in [13]. In the latter work, the method also accounts for perturbations with any structure, namely only single coefficients or blocks of the matrices can be perturbed while the others remain constant; in this paper we extend this algorithm in order to account for combined perturbations on the system matrices and the delay parameters.

The paper is organized as follows: in Section II we introduce the pseudospectral approach to the stability robustness analysis. The algorithm for the computation of the pseudospectral abscissa is described in Section III. In Section IV we illustrate the bisection-Newton's method implemented to find the distance to instability and in Section $\mathrm{V}$ we provide some numerical results.

\section{The Pseudospectral Approach}

The scope of this work is to evaluate the distance to instability of a dynamical system affected by real-valued structured perturbations on the coefficient matrices and by real-valued perturbations on the delay terms; considering 
system (1), the associated perturbed DEP reads as follows:

$$
M(\lambda) y=\left(\lambda I_{n}-\sum_{i=1}^{m}\left(A_{i}+B_{i} \delta A_{i} C_{i}\right) e^{-\lambda\left(\tau_{i}+\delta \tau_{i}\right)}\right) y=0
$$

where $y \in \mathbb{C}^{n}, \lambda \in \mathbb{C}, I_{n}$ is the identity matrix, $A_{i} \in$ $\mathbb{R}^{n \times n}, B_{i}, \delta A_{i}, \mathbb{C}_{i}$ are real-valued shape matrices of appropriate dimensions for all $i=1, \ldots, m, 0 \leq \tau_{1}<\cdots<\tau_{m}$ are the time-delays and $\delta \tau_{i} \in \mathbb{R}$ are such that $\left|\delta \tau_{i}\right|<\left|\tau_{i}\right|$ for all $i=1, \ldots, m$. Matrices $B_{i}, C_{i}$ define the structure of the perturbation on each matrix $A_{i}$ of the system; this allows us to perturb only a part of each matrix, e.g. a block or a single coefficient. Therefore the size of each matrix $\delta A_{i}$ may differ, i.e. $\delta A_{i} \in \mathbb{R}^{p_{i} \times q_{i}}, i=1, \ldots, m$. In the following, we will indicate the domain of matrices $\delta A_{i}$ as $\mathbb{R}^{*}:=\mathbb{R}^{p_{1} \times q_{1}} \times \cdots \times \mathbb{R}^{p_{m} \times q_{m}}$.

Since a distance measure is scalar, we need to define a scalar measure of the overall uncertainty, i.e., which takes into account all the perturbations from which the system is affected. Let us express $\Delta:=(\Delta A, \Delta \tau)=$ $\left(\delta A_{1}, \ldots, \delta A_{m}, \delta \tau_{1}, \ldots, \delta \tau_{m}\right) \in \mathbb{R}^{*} \times \mathbb{R}^{m}$ as the tuple containing the matrices perturbations $\delta A_{i}$ and the delays perturbations $\delta \tau_{i}$. Introducing the weights $w_{i}, v_{i} \in \mathbb{R}_{0}^{+} \cup$ $\{+\infty\}$ with $i=1, \ldots, m$, we define the following global norm

$$
\|\Delta\|_{\text {glob }}:=\left\|\left[\begin{array}{c}
w_{1}\left\|\delta A_{1}\right\|_{F} \\
\vdots \\
w_{m}\left\|\delta A_{m}\right\|_{F} \\
v_{1}\left|\delta \tau_{1}\right| \\
\vdots \\
v_{m}\left|\delta \tau_{m}\right|
\end{array}\right]\right\|_{\infty},
$$

where $\|\cdot\|_{F}$ stands for the matricial Frobenius norm. Therefore, an $\varepsilon$-bounded set of perturbation $\Delta$ is such that

$$
\left\|\delta A_{i}\right\|_{F} \leq \frac{\varepsilon}{w_{i}}, \quad\left|\delta \tau_{i}\right| \leq \frac{\varepsilon}{v_{i}}, i=1, \ldots, m
$$

Therefore, setting a weight $w_{i}=+\infty$ (or $\left.v_{i}=+\infty\right)$ implies that $\delta A_{i}=0$ (or $\delta \tau_{i}=0$ ) and that the relative nominal matrix (or delay) is assumed free from uncertainties. Given the above definition, for a fixed $\varepsilon$, we define the structured pseudospectrum of the perturbed DEP (2) as

$$
\Lambda_{\varepsilon}:=\bigcup_{\substack{\Delta \in \mathbb{R}^{*} \times \mathbb{R}^{m} \\\|\Delta\|_{\text {glob }} \leq \varepsilon}}\{\lambda \in \mathbb{C}: \operatorname{det} M(\lambda)=0\} .
$$

Given the notion of pseudospectrum, we introduce the pseudospectral abscissa function $\alpha_{\varepsilon}$, whose general definition reads as follows:

$$
\alpha_{\varepsilon}:=\sup \left\{\mathfrak{R}(\lambda): \lambda \in \Lambda_{\varepsilon}\right\}
$$

The expert reader, however, will observe that, from the well known properties of a DEP spectrum (see for instance Chapter 1 of [14]), the pseudospectral abscissa can also be defined as a maximum. From the definition of the pseudospectral abscissa $\alpha_{\varepsilon}$, the distance to instability easily follows as

$$
\inf \left\{\varepsilon: \alpha_{\varepsilon} \geq 0\right\} \text {. }
$$

Intuitively, we look for the minimum combined perturbation as defined in (3) that pushes one (or more) eigenvalues in the closed right-half of the complex plane. The method that we are going to illustrate in the following strongly relies on the next result about the continuity of the spectral abscissa, which is the real part of the rightmost point of a DEP (see again [14] for a proof of the result).

Theorem 1: Let $\alpha$ be the spectral abscissa of the DEP associated with system (1). Then the function $\alpha:\left(\mathbb{R}^{n \times n}\right)^{m} \times$ $\mathbb{R}^{m} \longrightarrow \mathbb{R}$

$$
\left(A_{1}, \ldots, A_{m}, \tau_{1}, \ldots, \tau_{m}\right) \longrightarrow \alpha\left(\left(A_{1}, \ldots, A_{m}, \tau_{1}, \ldots, \tau_{m}\right)\right)
$$

is continuous.

This result may seem trivial, but continuity w.r.t. the delay parameters does not hold when switching to neutral or algebraic delay differential equations: in these latter cases, the generated DEP is allowed to have a singular leading matrix and the spectral abscissa may not be continuous w.r.t. delay variations (see [15]).

As the pseudospectral abscissa is the maximum of a set of continuous function, it is also continuous w.r.t. to the system matrices $A_{1}, \ldots, A_{m}$ and the delay terms $\tau_{1}, \ldots, \tau_{m}$. Moreover, $\varepsilon_{1}<\varepsilon_{2}$ gives that $\Lambda_{\varepsilon_{1}} \subseteq \Lambda_{\varepsilon_{2}}$ (the set of allowable perturbations is enlarged); from the property of supremum function, the pseudospectral abscissa $\alpha_{\varepsilon}$ is therefore nondecreasing w.r.t. $\varepsilon$ (and in the generic case strictly increasing and differentiable around the zero crossing). We now have all the ingredients to redefine the distance to instability as the (unique) root of the equation

$$
\alpha_{\varepsilon}=0 \text {. }
$$

Therefore, our method aims at computing the real stability radius of system (1) as the root of this equation; to this purpose, we exploit an algorithm that computes the pseudospectral abscissa of DEP (2).

\section{An ITERATIVE METHOD TO COMPUTE THE PSEUdOSPECTRAL ABSCISSA}

The main idea of our algorithm is to generate a sequence of perturbed $\varepsilon$-bounded DEPs as in (2) such that the spectral abscissa of these problems is a monotonically increasing function, and the corresponding eigenvalue converges to the rightmost point of the pseudospectrum; as a matter of fact, the algorithm can be intepreted as a discretization of a gradient flow in the space of matrices perturbations and of delay terms pertubations induced by maximizing the real part of the rightmost eigenvalue. For what regards the perturbations on the system matrices, we exploit some low-rank properties of the optimal matrices perturbations, to which we will refer in the following as the matrices that generate the globally rightmost point of the pseudospectrum; analogously, we define the optimal time-delay perturbations. As we will see in the following, the behaviour of the derivative of the spectral 
abscissa w.r.t. these two different kinds of perturbations is comparable. In this work, we will make large use of the derivatives of eigenvalues w.r.t. different terms contained in the DEP; here we provide an easy reformulation of Lemma 2.7 in [16], where a general formula for these derivatives is given. In the following, we will then adapt this formula to our problem.

Lemma 2: Let $M(\lambda, \theta): \mathbb{C} \times \mathbb{C}^{d} \longrightarrow \mathbb{C}^{n \times n}$ be continuously differentiable, with $\lambda$ a simple eigenvalue whose corresponding left and right eigenvectors with unit norm are $x$ and $y$, and $\theta$ a set of parameters, then

$$
\frac{\partial \lambda}{\partial \theta_{i}}=-\frac{x^{*} \frac{\partial M(\lambda, \theta)}{\partial \theta_{i}} y}{x^{*} \frac{\partial M(\lambda, \theta)}{\partial \lambda} y}, \quad i=1, \ldots, d
$$

where $x^{*}$ is the conjugate transpose vector of $x$.

Our algorithm to compute the pseudospectral abscissa is based on the next fundamental result; this characterizes the optimal matrices perturbations and the optimal delay-terms perturbations of problem (2). Note that here and in the following, due to the symmetry of the (pseudo)spectrum of a DEP, we will refer to the globally rightmost point as the one in the upper half of complex plane.

Theorem 3: Let $\lambda_{\mathrm{RM}}$ be a globally rightmost point of the structured $\varepsilon$-pseudospectrum and assume it is a simple eigenvalue for some $\varepsilon$-bounded perturbation $\Delta$. Then

(i) There always exists a set of perturbations $\widetilde{\Delta A}=$ $\left(\widetilde{\delta A_{1}}, \ldots, \widehat{\delta A_{m}}\right)$, where $\widetilde{\delta A_{i}}$ has rank at most two and $w_{i}\left\|\widetilde{\delta A_{i}}\right\|_{F} \leq \varepsilon$ for all $i=1, \ldots, m$, and for which the rightmost eigenvalue is equal to $\lambda_{\mathrm{RM}}$;

(ii) Let $x, y$ be left and right eigenvectors of $\lambda_{\mathrm{RM}}$ respectively normalized such that $\|x\|=\|y\|=1$ and

$$
\xi:=x^{*}\left(I_{n}+\sum_{i=1}^{m}\left(A_{i}+B_{i} \delta A_{i} C_{i}\right) \tau_{i} e^{-\lambda\left(\tau_{i}+\delta \tau_{i}\right)}\right) y>0
$$

and let us define

$$
X=[\mathfrak{R}(x) \mathfrak{I}(x)], \quad Y=[\mathfrak{R}(y) \mathfrak{I}(y)],
$$

and

$$
\Gamma_{i}=\left[\begin{array}{cc}
\mathfrak{R}\left(e^{-\left(\tau_{i}+\delta \tau_{i}\right) \lambda_{\mathrm{RM}}}\right) & -\mathfrak{I}\left(e^{-\left(\tau_{i}+\delta \tau_{i}\right) \lambda_{\mathrm{RM}}}\right) \\
\mathfrak{I}\left(e^{-\left(\tau_{i}+\delta \tau_{i}\right) \lambda_{\mathrm{RM}}}\right) & \mathfrak{R}\left(e^{-\left(\tau_{i}+\delta \tau_{i}\right) \lambda_{\mathrm{RM}}}\right)
\end{array}\right]
$$

for $i=1, \ldots, m$. Then for each $i, B_{i}^{T} X \Gamma_{i} Y^{T} C_{i}^{T}$ can be either zero or nonzero. In the latter case, a particular set of optimal perturbations can be expressed as

$$
\widetilde{\delta A_{i}}=-\frac{\varepsilon}{w_{i}} \frac{B_{i}^{T} X \Gamma_{i} Y^{T} C_{i}^{T}}{\left\|B_{i}^{T} X \Gamma_{i} Y^{T} C_{i}^{T}\right\|_{F}}, i=1, \ldots, m
$$

(iii) Let $x, y$ be defined as before, then

$$
\frac{\partial \mathfrak{R}\left(\lambda_{\mathrm{RM}}\right)}{\partial \delta \tau_{i}}=\frac{1}{\xi} \mathfrak{R}\left(x^{*}\left(A_{i}+B_{i} \delta A_{i} C_{i}\right) \lambda e^{-\lambda\left(\tau_{i}+\delta \tau_{i}\right)} y\right)
$$

can also be either zero or nonzero. In the latter case, the optimal time-delay perturbations are such that

$$
\left|\widetilde{\delta \tau_{i}}\right|=\frac{\varepsilon}{v_{i}} .
$$

Proof: We do not report here the proof of the first part of the theorem; we refer the interested reader to Proposition 6.2 of [13]. The proof of the second part is the natural extension of the proof of Theorem 3.3 in [13] from unstructured to structured perturbations on system matrices where we included the uncertainties on the delay terms. Let us assume that $B_{i}^{T} X \Gamma_{i} Y^{T} C_{i}^{T}$ is a non-zero matrix for $i=1, \ldots, m$. Let us indicate with $\left(\delta A_{i}\right)_{s, t}$ the coefficient in position $(s, t)$ of matrix $\delta A_{i}$, and with $B_{i}^{(s)}, C_{i(t)}$ respectively the s-th column of $B_{i}$ and the t-th row of $C_{i}$, for $s=1, \ldots, p_{i}, t=$ $1, \ldots, q_{i}$. From Lemma 2, using some algebra manipulations we obtain that

$$
\begin{aligned}
& \frac{\partial \Re\left(\lambda_{\mathrm{RM}}\right)}{\partial \delta A_{i}}=\left(\frac{\partial \Re\left(\lambda_{\mathrm{RM}}\right)}{\partial\left(\delta A_{i}\right)_{s, t}}\right)_{\substack{s=1, \ldots, p_{i} \\
t=1, \ldots, q_{i}}}= \\
& =-\frac{1}{\xi} \mathfrak{R}\left(x^{*} B_{i}^{(s)} C_{i(t)} y e^{-\lambda\left(\tau_{i}+\delta \tau_{i}\right)}\right)_{\substack{s=1, \ldots, p_{i} \\
t=1, \ldots, q_{i}}}= \\
& =-\frac{1}{\xi}\left(B_{i}^{(s) T} \mathfrak{R}\left(x y^{*} e^{-\lambda\left(\tau_{i}+\delta \tau_{i}\right)}\right) C_{i(t)}^{T}\right)_{\substack{s=1, \ldots, p_{i} \\
t=1, \ldots, q_{i}}}^{T}= \\
& =-\frac{1}{\xi} B_{i}^{T} X \Gamma_{i} Y^{T} C_{i}^{T} \neq 0 .
\end{aligned}
$$

We want each $\delta A_{i}$ to be $\varepsilon$-bounded, so we impose the following constraints

$$
g_{i}:=\sum_{\substack{s=1, \ldots, p_{i} \\ t=1, \ldots, q_{i}}}\left(\delta A_{i}\right)_{s, t}^{2}-\frac{\varepsilon^{2}}{w_{i}^{2}} \leq 0, \quad i=1, \ldots, m .
$$

As $\lambda_{\mathrm{RM}}$ is the globally rightmost point of the pseudospectrum, from the theory of Lagrange multipliers we have that

$$
\frac{\partial \mathfrak{R}\left(\lambda_{\mathrm{RM}}\right)}{\partial \delta A_{i}}-2 \mu_{i} \delta A_{i}=0, \quad i=1, \ldots, m
$$

where $\mu_{i} \geq 0$ are the multipliers associated with the $g_{i}$ inequality constraints. Since $\frac{\partial \Re\left(\lambda_{\mathrm{RM}}\right)}{\partial \delta A_{i}}$ is a nonzero matrix, then $\mu_{i}$ is positive and $g_{i}$ is an active constraint; thus $\delta A_{i}$ is a positive multiple of $\frac{\partial \mathfrak{R}\left(\lambda_{\mathrm{RM}}\right)}{\partial \delta A_{i}}$ and in particular $\left\|\delta A_{i}\right\|_{F}=\frac{\varepsilon}{w_{i}}$ for $i=1, \ldots, m$, from which we derive the thesis. The proof of point (iii) can be easily deduced from the proof of point (ii) by applying the same reasoning to the perturbation $\delta \tau_{i}$ in the interval $\left[\tau_{i}-\varepsilon / v_{i}, \tau_{i}+\varepsilon / v_{i}\right], i=1, \ldots, m$; here we used the derivative in (8) which is obtained from Lemma 2.

This theorem provides useful information about the optimal matrices perturbations and the optimal delay-terms perturbations; in particular, we exploit the aforementioned lowrank properties for the matrices perturbations, so that we can restrict their research to the manifold

$$
\begin{aligned}
\widehat{\mathcal{S}}_{F}:=\left\{\left(\delta A_{1}, \ldots, \delta A_{m}\right) \in \mathbb{R}^{*}: \operatorname{rank}\left(\delta A_{i}\right) \leq 2,\right. \\
\left.w_{i}\left\|\delta A_{i}\right\|_{F} \leq \varepsilon, i=1, \ldots, m\right\} .
\end{aligned}
$$

In order to find the set of optimal perturbations $\widetilde{\Delta}:=$ $\widetilde{(\Delta A}, \widetilde{\Delta \tau})$, we define a continuous path in manifold $\widehat{\mathcal{S}}_{F}$ and one in the space of delay perturbations $\widehat{\mathcal{S}}_{\tau}:=\left[-\frac{\varepsilon}{v_{1}},+\frac{\varepsilon}{v_{1}}\right] \times$ $\cdots \times\left[-\frac{\varepsilon}{v_{m}},+\frac{\varepsilon}{v_{m}}\right]$; then our algorithm will perform a gradient 
flow discretization along these paths to converge to $\widetilde{\Delta}$. Let us first define the paths in a general way as follows

$$
\left\{\begin{array}{l}
\delta A_{i}(t)=-\frac{\varepsilon}{w_{i}} U_{i}(t) Q_{i}(t) V_{i}(t)^{T}, \quad t \in \mathbb{R}^{+} \\
\delta \tau_{i}(t)=\frac{\varepsilon}{v_{i}} q_{i}(t), \quad t \in \mathbb{R}^{+}
\end{array}\right.
$$

where the following properties need to be satisfied:

$$
\left\{\begin{array}{l}
U_{i}(t)^{T} \dot{U}_{i}(t)=0, \quad \forall t \geq 0 \\
V_{i}(t)^{T} \dot{V}_{i}(t)=0, \quad \forall t \geq 0 \\
\left\|Q_{i}(t)\right\|_{F} \leq 1, \quad \forall t \geq 0 \\
\left|q_{i}(t)\right| \leq 1, \quad \forall t \geq 0
\end{array}\right.
$$

and $U_{i}(t) \in \mathbb{R}^{p_{i} \times 2}, V_{i}(t) \in \mathbb{R}^{q_{i} \times 2}, Q_{i}(t) \in \mathbb{R}^{2 \times 2}$. The interested reader is referred to [13] for a detailed argumentation about the choice of the matrix decomposition. We now need to build matrices and functions with such properties. Let us introduce some additional matrices $R_{i}(t) \in \mathbb{R}^{p_{i} \times 2}, S_{i}(t) \in$ $\mathbb{R}^{q_{i} \times 2} M_{i}(t) \in \mathbb{R}^{2 \times 2}$ and functions $r_{i}(t)$ for $i=1, \ldots, m$ and let us indicate with $\langle A, B\rangle=\operatorname{Trace}\left(A^{T} B\right)$ the Frobenius inner product of two matrices: it is easy to prove that the solutions of the following differential equations (where we omit the parameter $t$ to simplify the notation)

$$
\left\{\begin{array}{l}
\dot{U}_{i}=\left(I_{n}-U_{i} U_{i}^{T}\right) R_{i}, \\
\dot{V}_{i}=\left(I_{n}-V_{i} V_{i}^{T}\right) S_{i}, \\
\dot{Q}_{i}= \begin{cases}M_{i}-\left\langle M_{i}, Q_{i}\right\rangle Q_{i}, & \text { if }\left\|Q_{i}\right\|_{F}=1,\left\langle M_{i}, Q_{i}\right\rangle>0 \\
M_{i}, & \text { otherwise, }\end{cases} \\
\dot{q}_{i}= \begin{cases}0 & \text { if }\left|q_{i}\right|=1, r_{i} q_{i}>0 \\
r_{i}, & \text { otherwise, }\end{cases}
\end{array}\right.
$$

satisfy the properties spelled out in (11) without losing of generality for any choice of $R_{i}(t), S_{i}(t), M_{i}(t), r_{i}(t)$; in practice, we look for matrices and functions with the desired properties as solutions of differential equations (12). Now, in order to construct some specific paths using equations (10), we need to make a choice on the arbitrary matrices $R_{i}(t), S_{i}(t), M_{i}(t)$ and functions $r_{i}(t)$. To this purpose, we use the derivative of an eigenvalue w.r.t. $t$ adapted from Lemma 2, where the eigenvalue problem $M(\lambda, t)$ is

$$
\left(\lambda I_{n}-\sum_{i=1}^{m}\left(A_{i}+B_{i} \delta A_{i}(t) C_{i}\right) e^{-\lambda\left(\tau_{i}+\delta \tau_{i}(t)\right)}\right) y=0,
$$

and the derivative reads as follows:

$$
\begin{aligned}
& \dot{\lambda}(t):=\frac{d \lambda(t)}{d t}= \\
& =\frac{\left.-x^{*}\left(\sum_{i=1}^{m} B_{i} \delta \dot{A}_{i}(t) C_{i} e^{-\lambda\left(\tau_{i}+\delta \tau_{i}(t)\right.}\right)\right) y}{\left.x^{*}\left(I_{n}+\sum_{i=1}^{m}\left(A_{i}+B_{i} \delta A_{i}(t) C_{i}\right) \tau_{i} e^{-\lambda\left(\tau_{i}+\delta \tau_{i}(t)\right.}\right)\right) y}+ \\
& +\frac{x^{*}\left(\sum_{i=1}^{m}\left(A_{i}+B_{i} \delta A_{i}(t) C_{i}\right) \lambda \delta \dot{\tau}_{i}(t) e^{-\lambda\left(\tau_{i}+\delta \tau_{i}(t)\right)}\right) y}{\left.x^{*}\left(I_{n}+\sum_{i=1}^{m}\left(A_{i}+B_{i} \delta A_{i}(t) C_{i}\right) \tau_{i} e^{-\lambda\left(\tau_{i}+\delta \tau_{i}(t)\right.}\right)\right) y} .
\end{aligned}
$$

By definition, each point in $\widehat{\mathcal{S}}_{F} \times \widehat{\mathcal{S}}_{\tau}$ corresponds to a perturbed DEP as in equation (13); we would like to build paths in $\widehat{\mathcal{S}}_{F}$ and $\widehat{\mathcal{S}}_{\tau}$ such that the spectral abscissa function of the corresponding DEPs is monotonically increasing. By means of the same normalization used in equation (6), we set the denominator in equation (14) equal to a positive real number $\xi$. Let us now consider for the sake of simplicity the case when $\dot{Q}_{i}(t)=M_{i}$ and $\dot{q}_{i}(t)=r_{i}$, and let us omit the dependence on $t$ in the notation, for sake of clarity, then we write

$$
\begin{aligned}
& \mathfrak{R}(\dot{\lambda})=\sum_{i=1}^{m} \frac{\varepsilon}{\xi w_{i}} \mathfrak{R}\left(x ^ { * } \left(B_{i} \dot{U}_{i} Q_{i} V_{i}^{T} C_{i}+B_{i} U_{i} \dot{Q}_{i} V_{i}^{T} C_{i}\right.\right. \\
& \left.\left.+B_{i} U_{i} Q_{i} \dot{V}_{i}^{T} C_{i}\right) e^{-\lambda\left(\tau_{i}+\delta \tau_{i}\right)} y\right)+ \\
& +\sum_{i=1}^{m} \frac{\varepsilon}{\xi v_{i}} \mathfrak{R}\left(x^{*}\left(A_{i}+B_{i} \delta A_{i} C_{i}\right) \lambda \dot{q}_{i} e^{-\lambda\left(\tau_{i}+\delta \tau_{i}\right)} y\right)= \\
& =\sum_{i=1}^{m} \frac{\varepsilon}{\xi w_{i}}\left\langle X, B_{i}\left(I_{n}-U_{i} U_{i}^{T}\right) R_{i} Q_{i} V_{i}^{T} C_{i} Y \Gamma_{i}^{T}\right\rangle+ \\
& +\sum_{i=1}^{m} \frac{\varepsilon}{\xi w_{i}}\left\langle X, B_{i} U_{i} M_{i} V_{i}^{T} C_{i} Y \Gamma_{i}^{T}\right\rangle+ \\
& +\sum_{i=1}^{m} \frac{\varepsilon}{\xi w_{i}}\left\langle X, B_{i} U_{i} Q_{i} S_{i}^{T}\left(I_{n}-V_{i} V_{i}^{T}\right) C_{i} Y \Gamma_{i}^{T}\right\rangle+ \\
& +\sum_{i=1}^{m} \frac{\varepsilon}{\xi v_{i}} \underbrace{\mathfrak{R}\left(x^{*}\left(A_{i}+B_{i} \delta A_{i} C_{i}\right) \lambda e^{-\lambda\left(\tau_{i}+\delta \tau_{i}\right)} y\right)}_{:=\beta_{i}} \dot{q}_{i}= \\
& =\sum_{i=1}^{m} \frac{\varepsilon}{\xi w_{i}}\left\langle B_{i}^{T} X \Gamma_{i} Y^{T} C_{i}^{T} V_{i} Q_{i}^{T},\left(I_{n}-U_{i} U_{i}^{T}\right) R_{i}\right\rangle+ \\
& +\sum_{i=1}^{m} \frac{\varepsilon}{\xi w_{i}}\left\langle U_{i}^{T} B_{i}^{T} X \Gamma_{i} Y^{T} C_{i}^{T} V_{i}, M_{i}\right\rangle+ \\
& +\sum_{i=1}^{m} \frac{\varepsilon}{\xi w_{i}}\left\langle S_{i},\left(I_{n}-V_{i} V_{i}^{T}\right) C_{i} Y \Gamma_{i}^{T} X^{T} B_{i} U_{i} Q_{i}\right\rangle+ \\
& +\sum_{i=1}^{m} \frac{\varepsilon}{\xi v_{i}} \beta_{i} \dot{q}_{i},
\end{aligned}
$$

and analogous expressions hold for the other cases in (12). Now, since $\left(I_{n}-U_{i} U_{i}^{T}\right),\left(I_{n}-V_{i} V_{i}^{T}\right)$ are positive semidefinite, the increasing monotonicity is guaranteed by the following choices

$$
\left\{\begin{array}{l}
\dot{U}_{i}=\left(I_{n}-U_{i} U_{i}^{T}\right) B_{i}^{T} X \Gamma_{i} Y^{T} C_{i}^{T} V_{i} Q_{i}^{T}, \\
\dot{V}_{i}=\left(I_{n}-V_{i} V_{i}^{T}\right) C_{i} Y \Gamma_{i}^{T} X^{T} B_{i} U_{i} Q_{i}, \\
\dot{Q}_{i}= \begin{cases}M_{i}-\left\langle M_{i}, Q_{i}\right\rangle Q_{i}, & \text { if }\left\|Q_{i}\right\|_{F}=1,\left\langle M_{i}, Q_{i}\right\rangle>0, \\
M_{i}, & \text { otherwise, }\end{cases} \\
\dot{q}_{i}= \begin{cases}0 & \text { if }\left|q_{i}\right|=1, \beta_{i} q_{i}>0 \\
\beta_{i}, & \text { otherwise, }\end{cases}
\end{array}\right.
$$

with $M_{i}=U_{i}^{T} B_{i}^{T} X \Gamma_{i} Y^{T} C_{i}^{T} V_{i}$. Given these choices for $\dot{U}_{i}, \dot{V}_{i}, \dot{Q}_{i}$ and $\dot{q}_{i}$, at each iteration of the algorithm we build a new perturbation of the original matrices and delay terms by moving in the ascent direction of the spectral abscissa w.r.t. parameter $t$; this is carried out by means of a forward Euler discretization of the differential equations (15) and then a projection of the new quantities onto the paths described in equation (10). Therefore, each iteration of our algorithm builds a new perturbed DEP like in (2) such that the sequence of the corresponding spectral abscissae is monotonically increasing. For the boundedness of the pseudospectrum, this sequence converges. We report here a short description in Algorithm 1.

Algorithm 1: Set perturbation size $\varepsilon$, weights $w_{i}, v_{i}$, stepsize $h$, tolerance $\eta$. Initialize $\left(\lambda^{(1)}, x^{(1)}, y^{(1)}\right)$, $\left(\tau_{1}^{(1)}, \ldots, \tau_{m}^{(1)}\right)$ and $U^{(1)}, V^{(1)}, Q^{(1)}, q^{(1)}$ and repeat for $k=1,2, \ldots$ 
1. Compute $X, Y, \Gamma_{i}$ as in Theorem 3 ;

2. Compute $\dot{U}_{i}^{(k)}, \dot{V}_{i}^{(k)}, \dot{Q}_{i}^{(k)}, \dot{q}_{i}^{(k)}$ using (15) for $i=$ $1, \ldots, m$

3. Compute for $i=1, \ldots, m$

$$
\left\{\begin{array}{l}
\widehat{U}_{i}^{(k+1)}=U_{i}^{(k)}+h \dot{U}_{i}^{(k)}, \\
\widehat{V}_{i}^{(k+1)}=V_{i}^{(k)}+h \dot{V}_{i}^{(k)}, \\
\widehat{Q}_{i}^{(k+1)}=Q_{i}^{(k)}+h \dot{Q}_{i}^{(k)}, \\
\widehat{\tau}_{i}^{(k+1)}=\tau_{i}^{(k)}+h \dot{q}_{i}^{(k)} ;
\end{array}\right.
$$

4. Project $\widehat{U}_{i}^{(k+1)}, \widehat{V}_{i}^{(k+1)}, \widehat{Q}_{i}^{(k+1)}$ onto manifold $\widehat{\mathcal{S}}_{F}$ : use a compact $\mathrm{QR}$ decomposition to normalize $\widehat{U}_{i}^{(k+1)}, \widehat{V}_{i}^{(k+1)}$, and, if $\left\|\widehat{Q}_{i}^{(k+1)}\right\|_{F}>1$, also normalize it;

5. Possibly rescale $\widehat{\tau}_{i}^{(k+1)}$ to an $\varepsilon$-bounded set of delay terms:

$$
\left\{\begin{array}{l}
\tau_{i}^{(k+1)}=\min \left(\tau_{i}+\frac{\varepsilon}{v_{i}}, \widehat{\tau}_{i}^{(k+1)}\right), \text { if } \dot{q}_{i}^{(k)} \geq 0 \\
\tau_{i}^{(k+1)}=\max \left(0, \tau_{i}-\frac{\varepsilon}{v_{i}}, \widehat{\tau}_{i}^{(k+1)}\right), \text { if } \dot{q}_{i}^{(k)}<0
\end{array}\right.
$$

6. Define $\delta A_{i}=-\frac{\varepsilon}{w_{i}} U_{i}^{(k+1)} Q_{i}^{(k+1)} V_{i}^{(k+1) T}$ and $\tau_{i}+$ $\delta \tau_{i}=\tau_{i}^{(k+1)}$ and compute the rightmost eigenvalue $\lambda$ and the normalized eigenvectors $x, y$ of the DEP (2);

7. Stop the algorithm if this condition holds

$$
\mathfrak{R}(\dot{\lambda})<\eta
$$

otherwise start again from step 1;

8. Return the pseudospectral abscissa $\mathfrak{R}(\lambda)$.

In step 6 we need to solve DEPs: this is carried out using the algorithm and software described in [15]. For major details about the initialization we refer the reader to [13]: basically, $\delta A_{i}$ are initialized performing one step of a fixed point iteration whose iterate function is defined in (7), while $\delta \tau_{i}$ are simply initialized following the derivative in Equation (8). Note that discretized gradient flow in Algorithm 1 can also be interpreted as the application of a first order optimization method (gradient search). Furthermore, due to space limitation we have only sketched the basic algorithm excluding the adaptive stepsize strategy in our implementation, which has an interpretation in terms of a globalization strategy. As a consequence, under standard conditions convergence to perturbations satisfying the first order necessary optimality conditions can be guaranteed. As we have to compute the globally rightmost point of the pseudospectrum, we use the strategy from [13], which is based on generating initial conditions from several rightmost eigenvalues. Finally, the previous algorithm also allows to include constraints on the dependence among matrices perturbations $\delta A_{i}$. For instance, we can impose some perturbations $\delta A_{i}$ to be equal to each others; an example is given in the cutting process in Section V.

\section{A Bisection-NeWTON'S METHOD FOR THE DISTANCE TO INSTABILITY}

As we discussed in Section II, under mild conditions we can compute the distance to instability of system (2) as the value of $\varepsilon$ corresponding to the unique root of the equation $\alpha_{\varepsilon}=0$, where $\alpha_{\varepsilon}$ is the pseudospectral abscissa computed using the method in Section III. In order to use Newton's method to solve the equation, at least almost differentiability of the function is required. The pseudospectral abscissa function $\alpha_{\varepsilon}$ is by definition a real-valued increasing monotone function of $\varepsilon$ and bounded on any interval $\left[\varepsilon_{L}, \varepsilon_{R}\right]$, thus it is a function of bounded variation; from the Lebesgue's differentiation theorem of functions of bounded variation (see for instance Theorem 3.4 of [17]) we can conclude that $\alpha_{\varepsilon}$ is almost everywhere differentiable w.r.t. $\varepsilon$; furthermore, it is differentiable whenever the rightmost eigenvalue corresponding to the optimal perturbations is simple. The next theorem then provides an explicit expression for the derivative.

Theorem 4: Let $\left(\lambda_{\mathrm{RM}}(\varepsilon), x(\varepsilon), y(\varepsilon)\right)$ be respectively the globally rightmost point of $\Lambda_{\varepsilon}$ and its left and right normalized eigenvectors; let also $\widehat{\Delta A}(\varepsilon)=\left(\widetilde{\delta A_{1}}(\varepsilon), \ldots, \widetilde{\delta A_{m}}(\varepsilon)\right)$ and $\widetilde{\Delta \tau}(\varepsilon)=\left(\widetilde{\delta \tau_{1}}(\varepsilon), \ldots, \widetilde{\delta \tau_{m}}(\varepsilon)\right)$ be functions such that $\lambda_{\mathrm{RM}}(\varepsilon)$ is the unique rightmost eigenvalue of system (2) with $\delta A_{i}=\widetilde{\delta A_{i}}(\varepsilon)$ and $\delta \tau_{i}=\widetilde{\delta \tau_{i}}(\varepsilon)$ and

$$
\widetilde{\delta A_{i}}(\varepsilon)=-\frac{\varepsilon}{w_{i}} \widetilde{U_{i}}(\varepsilon) \widetilde{Q_{i}}(\varepsilon) \widetilde{V}_{i}(\varepsilon)^{T} \text { and } \widetilde{\delta \tau_{i}}(\varepsilon)=\frac{\varepsilon}{v_{i}} \widetilde{q}_{i}(\varepsilon)
$$

for $i=1, \ldots, m$; then denoting

$\zeta:=x^{*}\left(I_{n}+\sum_{i=1}^{m}\left(A_{i}+B_{i} \widetilde{\delta A_{i}} C_{i}\right) e^{\left(-\lambda_{\mathrm{RM}}\left(\tau_{i}+\widetilde{\delta \tau_{i}}\right)\right)}\left(\tau_{i}+\widetilde{\delta \tau_{i}}\right)\right) y$

we can express the derivative of the pseudospectral abscissa w.r.t $\varepsilon$ as follows

$$
\begin{aligned}
& \frac{\partial \alpha_{\varepsilon}}{\partial \varepsilon}=\frac{\partial \mathfrak{R}\left(\lambda_{\mathrm{RM}}\right)}{\partial \varepsilon}= \\
& -\frac{1}{\zeta} \mathfrak{R}\left[x^{*}\left(\sum_{i=1}^{m} B_{i} \frac{\partial \widetilde{\delta A_{i}}}{\partial \varepsilon} C_{i} e^{\left(-\lambda_{\mathrm{RM}}\left(\tau_{i}+\widetilde{\delta \tau_{i}}\right)\right)}\right) y+\right. \\
& \left.+x^{*}\left(\sum_{i=1}^{m}\left(A_{i}+B_{i} \widetilde{\delta A_{i}} C_{i}\right) e^{\left(-\lambda_{\mathrm{RM}}\left(\tau_{i}+\widetilde{\delta \tau_{i}}\right)\right)} \lambda_{\mathrm{RM}} \frac{\partial \widetilde{\delta \tau_{i}}}{\partial \varepsilon}\right) y\right]= \\
& +\frac{1}{\zeta} \mathfrak{R}\left[x ^ { * } \left(\sum_{i=1}^{m} \frac{1}{w_{i}} B_{i} \widetilde{U_{i}} \widetilde{Q_{i}} \widetilde{V}_{i}^{T} C_{i} e^{\left(-\lambda_{\mathrm{RM}}\left(\tau_{i}+\frac{\varepsilon}{v_{i}} \widetilde{q_{i}}\right)\right)}+\right.\right. \\
& \left.\left.+\sum_{i=1}^{m}\left(A_{i}-\frac{\varepsilon}{w_{i}} B_{i} \widetilde{U_{i}} \widetilde{Q_{i}} \widetilde{V}_{i}^{T} C_{i}\right) e^{\left(-\lambda_{\mathrm{RM}}\left(\tau_{i}+\frac{\varepsilon}{v_{i}} \widetilde{q_{i}}\right)\right)} \lambda_{\mathrm{RM}} \frac{\widetilde{q_{i}}}{v_{i}}\right) y\right],
\end{aligned}
$$

where we omit the dependence on $\varepsilon$ to simplify the notation. Observe that we obtain the last expression because we are dealing with optimal combined perturbations at every fixed $\varepsilon$.

This formula is again derived from Lemma 2, so we will not provide a proof. As Newton's method is a local method, we first look for a good starting value that guarantees its convergence. This is carried out by performing some iterations of the bisection method, that we repeat until we find an $\varepsilon$ that generates a pseudospectral abscissa close enough to 0 . We now have all the ingredients needed to apply Newton's method to our problem; the basic iteration is shortly described in the following Algorithm 2. 
Algorithm 2: Initialize perturbation $\varepsilon_{1}$ and repeat for $k=$ $1,2, \ldots$ until convergence

1. Compute the pseudospectral abscissa $\alpha_{\varepsilon_{k}}$ of DEP (2);

2. Update

$$
\varepsilon_{k+1}=\varepsilon_{k}-\frac{\alpha_{\varepsilon_{k}}}{\left.\frac{\partial \alpha_{\varepsilon_{k}}}{\partial \varepsilon}\right|_{\varepsilon=\varepsilon_{k}}} .
$$

\section{NUMERICAL EXPERIMENTS}

In this section we want to demonstrate the efficiency of both the algorithms here presented. First we use an example from [18] to show how the pseudospectral abscissa behaves by taking into account perturbations on the system matrices and on the delay terms, and in particular we compare the level sets of the pseudospectral abscissa and of the spectral abscissa; then we provide a benchmark of examples for which we computed the distance to instability.

Example 1: We consider a simplified version of the mechanical rotational cutting process presented in [18] and [19], where the delay term $\tau$ is constant in time; its characteristic equation reads

$$
\lambda^{2}+2 \xi \omega \lambda+\omega^{2}+\frac{k}{m}\left(1-e^{-\lambda \tau}\right)=0
$$

where $\omega$ is the natural frequency, $\xi$ the damping ratio, $m$ the modal mass and $k$ the cutting force coefficient and it is associated with the DEP with matrices

$$
A_{1}=\left[\begin{array}{cr}
0 & 1 \\
-\omega^{2}-k / m & -2 \xi \omega
\end{array}\right], A_{2}=\left[\begin{array}{cc}
0 & 0 \\
k / m & 0
\end{array}\right],
$$

and $\tau_{1}=0, \tau_{2}:=\tau$. We want to consider structured perturbations on the delay $\tau$ and on the coefficient $k$, so our perturbed DEP looks like system (2) where we set $B_{1}=\left[\begin{array}{ll}0 & 1 / m\end{array}\right]^{T}, B_{2}=-B_{1}, C_{1}=\left[\begin{array}{ll}1 & 0\end{array}\right], C_{2}=C_{1}$ and $\delta A_{1}=\delta A_{2}$ is the scalar perturbation of parameter $k$. We want $|\delta k| \leq 1.5 * 10^{6},|\delta \tau| \leq 1.5 * 10^{-3}$, thus we set $\varepsilon=0.1, v_{1}=+\infty, v_{2}=0.015^{-1}, w_{1}=w_{2}=$ $\left(1.5 \cdot 10^{7}\right)^{-1}$. In Fig. 1 we plot on the $k-\tau$ plane the level sets equal to 0 of the spectral abscissa (blue line) and of the pseudospectral abscissa for $\varepsilon=0.1$ (red line); we observe that each point on the pseudospectral abscissa level set is connected with a point on the spectral abscissa level set by means of an $\varepsilon$-bounded $\widetilde{\Delta}$ perturbation. The blocks built on a few points of the pseudospectral abscissa level set represent the area spanned in the $k-\tau$ plane by all the possible $\varepsilon$-bounded perturbations; it is easy to see that $\widetilde{\Delta}$ is often characterized by a maximum norm perturbation on the parameter $k$, while this does not always happen when considering perturbations on delay terms. For this reason we observe an occasionally flat nature of the pseudospectral abscissa level set. However, the latter behaviour can also be observed w.r.t. to matrices coefficients: as a matter of fact, the pseudospectral abscissa w.r.t. to structured system matrices perturbations is not always generated by a maximum-norm perturbation; the interested reader can refer to [13] for an example. Finally, note that if the above computations are repeated for $w_{1}=w_{2}=+\infty$, then the algorithms returs the delay margin.

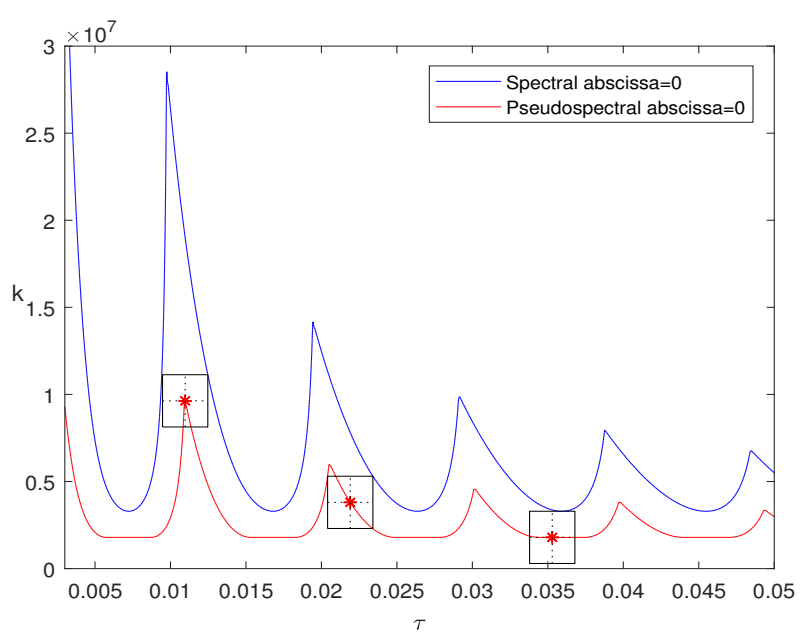

Fig. 1. Rectangular blocks represent an $\varepsilon$-bounded set of perturbations for the point marked with a red asterisk; the perturbation has maximum size $\frac{\varepsilon}{w_{i}}$ on the parameter $k$ and $\frac{\varepsilon}{v_{i}}$ on the delay $\tau$.

TABLE I

THE TABLE SHOWS THE DISTANCE TO INSTABILITY $\varepsilon_{r}$ FOR PROBLEMS IN THE BENCHMARK

\begin{tabular}{ccccc}
\hline Problem & $(n, m)$ & $\varepsilon_{r}$ & $\alpha_{\varepsilon_{r}}$ & $\#$ it \\
\hline 1 & $(3,2)$ & $1.112792276 \mathrm{e}-02$ & $-2.663325696 \mathrm{e}-17$ & 6 \\
2 & $(3,2)$ & $5.600882011 \mathrm{e}-01$ & $2.753544234 \mathrm{e}-15$ & 4 \\
3 & $(5,6)$ & $9.400004821 \mathrm{e}-01$ & $6.365699553 \mathrm{e}-09$ & 30 \\
4 & $(5,6)$ & $1.459273837 \mathrm{e}-01$ & $4.107825191 \mathrm{e}-16$ & 6 \\
5 & $(10,3)$ & $2.352096503 \mathrm{e}-03$ & $-5.969697629 \mathrm{e}-16$ & 4 \\
6 & $(10,3)$ & $9.139676624 \mathrm{e}-02$ & $-5.520865458 \mathrm{e}-16$ & 5 \\
7 & $(20,3)$ & $1.041499774 \mathrm{e}-01$ & $1.000000000 \mathrm{e}-15$ & 5 \\
8 & $(30,3)$ & $1.013715072 \mathrm{e}-01$ & $-9.059148660 \mathrm{e}-16$ & 5 \\
\hline
\end{tabular}

Results of the bisection-Newton's method to compute the distance to instability are reported in Table I. We created a collection of problems from the literature where we have perturbed physical parameters appearing in system matrices and delay terms ${ }^{1}$. For all of these problems we ran the algorithm multiple times considering different perturbations, so they appear more than once in Table I; problems 1 and 2 are taken from [20], problems 3 and 4 are from [21]. Problems 5-8 are obtained from [22] as a discretization of a partial delay-differential equation, for which we adopt different refinements of the discretization. Details about the uncertainties considered in each problem are also included in the benchmark ${ }^{1}$. In Table I, the first column refers to the numbering of the problems in the benchmark, the second column displays the dimension of the problem and the number of delays, the third and fourth columns show respectively the distance to instability $\varepsilon_{r}$ and the corresponding pseudospectral abscissa $\alpha_{\varepsilon_{r}}$ and the last column shows the number of Newton's method iterations needed for convergence.

\footnotetext{
${ }^{1}$ The collection is available at the webpage http://twr.cs.kuleuven.be/research/software/delay-control/benchmarksdistance-instability.zip
} 


\section{CONCLUDING REMARKS}

We presented an algorithm that computes the pseudospectral abscissa of a delay eigenvalue problem with a nonsingular leading matrix, taking into account real-valued structured perturbations on the system matrices and perturbations on the delay terms. This is a local method, in the sense that the convergence conditions imposed on the algorithm also apply for locally but not globally rightmost points of the pseudospectrum; however, in practice, we have seldom observed this behaviour, and using different starting values has proved to be a good strategy to guarantee convergence to the globally rightmost point. This algorithm has then been extended to compute the distance to instability (the actual value, i.e. no possibly conservative lower or upper bounds) of delay eigenvalue problems using a bisection-Newton's method, which has turned out to be very fast in convergence. Thanks to the low-rank dynamics of optimal perturbations exploited in the computation of the pseudospectral abscissa and to its iterative nature, the method has also potential for sparse large-scale systems.

\section{REFERENCES}

[1] Y. Genin, R. Stefan, and P. Van Dooren, "Real and complex stability radii of polynomial matrices," Linear Algebra and its Applications, vol. 351-352, pp. 381-410, 2002.

[2] J. Sreedhar, P. Van Dooren, and A. Tits, "A fast algorithm to compute the real structured stability radius," in Stability Theory - Hurwitz Centenary Conference, ser. International Series of Numerical Mathematics, R. Jeltsch and M. Mansour, Eds. Birkhäuser, 1996, vol. 121, pp. 219230.

[3] P. Van Dooren and V. Vermaut, "On stability radii of generalized eigenvalue problems," in Proceedings of the 1997 European Control Conference (ECC'97), Brussels, Belgium, 1997.

[4] J. Chen, G. Gu, and C. A. Nett, "A new method for computing delay margins for stability of linear delay systems." Systems \& Control Letters, vol. 26, pp. 107-117, 1995.

[5] S.-I. Niculescu, "On some stability regions of a multiple delays linear systems with applications," in Proceedings of the 15th IFAC World Congress, Barcelona, Spain, 2002.

[6] J. Chen and H. A. Latchman, "Frequency sweeping tests for stability independent of delay." Journal of Mathematical Analysis and Applications, vol. 40, pp. 1640-1645, 1995.

[7] W. Michiels and S.-I. Niculescu, "Characterization of delayindependent stability and delay-interference phenomena," SIAM Journal on Control and Optimization, vol. 45, no. 6, pp. 2138-2155, 2007.

[8] S. Niculescu, Delay effects on stability. A robust control approach, ser Lecture Notes in Control and Information Sciences. Springer-Verlag, 2001, vol. 269.

[9] J. Chen and S. Niculescu, "Robust stability of quasi-polynomials: Frequency-sweeping conditions and vertex tests," IEEE Transactions on Automatic Control, vol. 53, pp. 1219-34, 2008.

[10] W. Michiels, E. Fridman, and S. Niculescu, Robustness assessment via stability radii in delay parameters. International Journal of Robust and Nonlinear Control, 2009, in press (published on-line).

[11] N. Guglielmi and C. Lubich, "Low-rank dynamics for computing extremal points of real pseudospectra," SIAM Journal of Matrix Analysis and Applications, vol. 34, pp. 40-66, 2013.

[12] W. Michiels and N. Guglielmi, "An iterative method for computing the pseudospectral abscissa for a class of nonlinear eigenvalue problems," SIAM Journal on Scientific Computing, vol. 34, no. 4, pp. A2366A2393, 2012.

[13] F. Borgioli, W. Michiels, and N. Guglielmi, "Characterizing and computing the real pseudospectral abscissa for nonlinear eigenvalue problems," 2017, submitted for publication to Numerische Mathematik, available from https://lirias.kuleuven.be/handle/123456789/548090.

[14] W. Michiels and S. Niculescu, Stability and stabilization of time-delay systems. An eigenvalue based approach. SIAM, 2007.
[15] W. Michiels, "Spectrum based stability analysis and stabilization of systems described by delay differential algebraic equations," IET Control Theory and Applications, vol. 5, no. 16, pp. 1829-1842, 2011.

[16] K. Schreiber, "Nonlinear eigenvalue problems: Newton-type methods and nonlinear rayleigh functionals," Ph.D. dissertation, TU Berlin, 2008.

[17] E. Stein and R. Shakarchi, Real Analysis, ser. Springer Series in Operations Research. Princeton University Press, 2005.

[18] W. Michiels, V. Van Assche, and S. Niculescu, "Stabilization of timedelay systems with a controlled, time-varying delay and applications," IEEE Transactions on Automatic Control, vol. 50, no. 4, pp. 493-504, 2005.

[19] S. Jayaram, S. Kapoor, and R. DeVor, "Analytical stability analysis of variable spindle speed machines," Journal of Manufacturing and Engineering, vol. 122, pp. 391-397, 2000.

[20] T. Wagenknecht, W. Michiels, and K. Green, "Structured pseudospectra for nonlinear eigenvalue problems," Journal of Computational and Applied Mathematics, vol. 212, no. 2, pp. 245-259, 2006.

[21] Vyhlídal, T., "Analysis and synthesis of time delay system spectrum," Ph.D. dissertation, Department of Mechanical Engineering, Chech Technical University at Prague, 2003.

[22] Jarlebring, E., "The spectrum of delay-differential equations: numerical methods, stability and perturbation," Ph.D. dissertation, TU Braunschweig, Institut Computational Mathematics, 2008. 